\title{
Beobachtungen über die Wirkung ins Auge eingedrungener Mretallsplitter.
}

\author{
Von \\ Professor Th. Leber \\ in Göttingen.
}

Die Wirkungen in's Auge eingedrungener Fremdkörper habe ich in den letzten Jahren durch zahlreiche Versuche an Thieren genauer festzustellen gesucht, besonders mit Rücksicht auf die Frage, wie weit diese Fremdkörper an und für sich Entzündung bewirken und wie weit die letztere durch Verunreinigung des Fremdkörpers mit niederen Organismen entsteht. Ich habe über die Ergebnisse dieser Versuche bisher nur einige kurze Mittheilungen gemacht*), da ich beabsichtige, dieselben später im Zusammenhang ausführlicher zu veröffentlichen. Auch jetzt möchte ich auf diesen Gegenstand nur kurz eingehen; doch veranlasst mich die vorhergehende Arbeit Dr. Franke's zu

*) Vergl. Th, Leber, Ueber die Wirkung von Fremdkörpern in Inneren des Auges. Transact. of the internat. med. Congress. VII. Sess. Vol. III, p. 15-19. London 1881. - Landmann, Ueber die Wirkung aseptisch in das Auge eingedrungener Fremdkörper. Dieses Archiv XXVIU. 2, S. 155-156. - Th. Leber, Notiz über die Wirkung metallischer Fremdkörper im Innern des Auges. Ibid. XXVIII. 2, S. 237-238. 1882. 
einigen Mittheilungen, zumal ich über einige kürzlich beobachtete Krankheitsfulle berichten kann, welche die reine, uncomplicirte Wirkung in das Auge eingedrungener Metallsplitter mit ziemlicher Sicherheit festzustellen erlaubten.

Die Resultate der Thierversuche dürfen natürlich nur mit Vorsicht auf den Menschen ubertragen werden. Wenn man auch principielle Abweichungen wohl nicht zu erwarten hat, so sind doch in Bezug auf den Grad der durch verschiedene Fremdkörper bewirkten entzündlichen Reaction Unterschiede sehr wohl denkbar.

Einfach liegt die Sache fur die chemisch indifferenten Fremdkörper. Dass deren blosse Anwesenheit im Auge keine Entzündung bewirkt, wird nicht nur durch Versuche an Thieren, sondern auch durch zahlreiche Beobachtungen am Menschen, von denen noch kürzlich Landmann eine fleissige Zusammenstellung*) geliefert hat, zur Genüge beweisen, so dass wir die in einzelnen Füllen gleichwohl auftretende Entzündung gewiss auf die Mitwirkung anderer Ursachen zu schieben berechtigt sind.

Weit schwieriger ist die Beurtheilung der viel häufigeren Verletzungen durch chemisch nicht indifferente Fremdkörper, insbesondere Eisen, resp. Stahl, und Kupfer, wo es gewöhnlich zum Auftreten von Entzündung kommt, von der wir aber bei der Ungewissheit, ob der Fremdkörper aseptisch war oder nicht, im einzelnen Fall nicht sicher angeben kömnen, $a b$ sie der Wirkung des Fremdkörpers selbst oder einer septischen Verunreinigung desselben zuzuschreiben ist. Es ist bekannt und ergibt sich auch wieder aus der in diesem Heft enthaltenen sorgfültigen Zusammenstellung Franke's von Fremdkörpern in der vorderen Augenkammer, dass die Wirkungen derselben auch unter scheinbar gleichen Bedingungen keineswegs constant sind, indem ihrem Eindringen bald stärkere, bald schwächere

*) loc. cit. 
Entzündung folgt und indem in seltenen Fällen wach geringfügiger Reizung sogar eine Toleranz für den Fremdkörper eintritt, der sodann lange Zeit ohne Schaden vertragen werden kann.

Auf den ersten Blick könnte es am einfachsten scheinen, diese Differenzen durch die Annahme zu erklären, dass das Eintreten von Entzündung, abgesehen von ganz geringen Graden derselben, auch bei Fremdkörpern aus Eisen und Kupfer immer durch eine gleichzeitige Infection bedingt sei, während das unschädliche Verweilen im Auge auf der zufällig aseptischen Beschaffenheit des Fremdkörpers beruhe. Gegen diese Annahme lässt sich aber schon einwenden, dass bei der Hänfigkeit, mit welcher auf das Eindringen der genannten Metalle entzündliche Erscheinungen folgen, die septisch complicirten Verletzungen dieser Art die Regel, die reinen dagegen die seltene Ausnahme bilden müssten, was doch nicht gerade wahrscheinlich ist. Ueberdies haben die Versuche gezeigt, dass von den genannten Metallen bei Thieren wenigstens das Kupfer, auch bei vollig aseptiseher Einführung, unter gewissen Bedingungen eitrige Entzündung hervorrufen kann. Es haben sich dabei auch bemerkenswerthe Verschiedenheiten, nicht nur zwischen der Wirkung von Kupfer und Eisen, sondern auch je nach der Lage des liremdkörpers in Bezug auf die gefässhaltigen Theile des Auges, herausgestellt, woraus hervorgeht, wie zahlreich und complicirt die hier in Betracht zu ziehenden ursächlichen Momente sind. Wir werden daher, um zur richtigen Einsicht in die Vorgänge beim menschlichen Auge zu gelangen, von den sicher gestellten Ergebnissen der Thierversuche auszugehen und die Erscheinungen möglichst genau untersuchter Fälle von Verletzungen beim Menschen damit zu vergleichen haben. Hierzu möchte ich mit diesen Zeilen einen kleinen Beitrag liefern. 
Ich will runächst die in meinen Versuchen whrm genommenen Folgen des Eindringens aseptischer Fremdkörper aus Eisen und Kupfer in die vordere Augenkammer kurz zusammenfassen.

Bringt man Stücke von Nähnadeln, die man zuvor durch Erhitzen desinficirt hat, in die vordere Kammer vom Kaninchen, indem man sie mit einer geeigneten Pincette einsticht and mit einer stumpfen Nadel von gleicher Dicke hineinstösst, so treten auch bei beträchtlicher Grösse des Wremdkörpers nur sehr unbedeutende Reizerscheinungen auf. Dieselben sind naturilich um so geringer, je glatter die Einführung gelingt und je weniger Kammerwasser dabei abgeflossen ist. Der Fremdkörper senkt sich in den wuteren Theil der vorderen Kammer; es zeigt sich nur eine geringfingige Injection, gewöhnlich auf den unteren Umfang der Hornhaut beschränkt, die sich sogar sehr bald für einige Zeit ganz verliert, um später wieder anf's neue hervorzutreten, besonders bei Lageveränderungen des spitzen Fremalkörpers, die aber immer nur ganz geringen Grades ist. Nach einigen Tagen bekommt die Nadel zuerst an ihrem dickeren Ende, dann auch an anderen Stellen, durch Auflagernng einer dunnen Exsudatsehicht eine gelbliche Färbung, die allmählich in eine dunklere, rostbraune Farbe übergeht; ist diese Auflagerung nur partiell, so verliert die Nadel an den anderen Stellen wenigstens ihren Metallglanz. Das Kammerwasser zeigt keine Trübung und keine uber die nächste Umgebung der Nadel hinausgehende Exsudation. An deu Stellen, wo die Nadel mit der Hornhant oder Iris in Berührung ist, wird sie gewöhnlich mit diesen Theilen durch eine geringe Menge braungefärbten Exsudates verklebt; der anstossende Theil der Hornhaut zeigt mitunter" eine leichte, ganz umschriebene Trübung oder wird Sitz einer zarten Vascularisation. Kommt die Nadelspitze gegen die Hornhaut zu stehen, so wird diese allmählich usurirt und die Nadel kommt an der Aussen- 
fläche der Hornhaut zum Vorschein. Ich habe in mehreren Fällen solche Augen, die sich dabei vollkommen wohl befanden, Monate und Jahre lang beobachtet, ohne dass andere Folgezustände auftraten; einmal wurde nach 527 Tagen das hervorragende Ende mit der Pincette extrahirt, worauf sehr rasch vollkommene Heilung erfolgte.

Ganz anders ist das Verbalten bei gleich grossen and gleich gelagerten Kupferstücken. Sassen dieselben der Iris auf, so sah ich ansnahmslos schon nach wenigen Tagen unter viel stärkerer Ciliarinjection und sebr bedeutender Irishyperämie den Fremdkörper von Eiter eingehullt werden, der nach und nach einen ziemlich grossen Theil der vorderen Kammer erfüllte. Merkwürdig ist dabei die Art der Begrenzung des Eiters, der mit einer scharf begrenzten Kuppe nach oben in die Kammer vorragt und sich in Absätzen allmählich weiter vorsehiebt; man erhält dabei den Eindruck, dass der Eiter ausschliesslich von der nächsten Umgebung des Fremdkörpers geliefert werde. Später kommt die Eiterproduction mehr zum Stillstand, das Exsudat schrumpft und kann als eingedickte Masse den Fremdkörper gewissermassen abkapseln. Die daror liegende Hornhant wird mehr oder minder stark infiltrirt oder mit der Zeit zuweilen local erweicht, so dass es zur spontanen Ausstossung des Kupferstückchens kommen kann, worauf trotz dieser so schweren Entzündung noch eine rasche und relativ gute Heilung eintritt. Immer bleibt die Entzündung auf den rorderen Theil des Auges und auf die nähere Umgebung des Fremdkörpers beschränkt, nie habe ich sie, wie bei infectiösen Entzündungen, einen propagatorischen Charakter annehmen sehen.

Diese Eigenthümlichkeit sowohl, als auch die Constanz der Erscheinungen bestätigt durchaus meine Annahme, dass die hier beobachtete Entzündung der chemischen Wirkung des Kupfers und nicht einer, übrigens auch durch 
die Versuchsinethode sicher auszuschljessenden, zufalligen Infection zuzuschreiben ist.

Von besonderem Interesse sind die Erscheinungen, welche man nach Einführung zahlreicher feiner Partikelchen der genannten Metalle in die vordere Kammer beobachtet, welche ich hier nur in aller Kürze erwähnen will. Nach Injection von Kupferfeile in die vordere Kammer ungiebt sich jedes der zahlreichen Partikelchen mit einer Eiterhulle, so dass die Iris von oiner zahllosen Menge feinster Eiterknötchen bedeckt ist. Nach einiger Zoit tritt eine langsame Abnahme dieser Eiterknötchen ein, dieselben verschwinden aJlmalig spurlos und meistens ohne dass dabei das eingeschlossene Metallpartikelchen zum Vorschein lommt. Der Endansgang ist vollständige Restitutio ad integrum; ich habe in der That gesehen, dass sämmtliche eingeführte Metallpartikelchen spulos resorbirt wurden.

Un die Wirkung des Eisens damit zu vergleichen, wurde an zwei Augen eine Suspension von Ferrum hydrogenio reductum in die vordere Kammer injieirt. Trotzdem wegen der feineren Vertheilung eine noch stärkere Wirkung zu erwarten war, erfolgte nur geringe Reaction. Der Metallstaub wurde in ein die Prpille deckendes Fibringerinnsel eingeschlossen, das sich ziemlich rasch resorbirte und nach einigen Wochen grösstentheils verscbwunden war. Am Tage der Injection waren in dem Fibrin noch zahlreiche grau-metallisch aussehende Pünktchen zu sehen, während man in den nächsten Tagen statt ibrer feinste gelbliche Pünktchen wahrnahm. Hierin zeigt sich offenbar eine Analogie zu dem Verhalten des Kupfers, doch mit dem Unterschied, dass hier die entzündungserregende Wirkung nur eine äusserst geringe ist.

Im grellen Contrast zu den Folgen, welche Kupferdrähte hervorrufen, wenn sie der Iris aufgelagert sind, steht das völlige Ausbleiben von Entzündung, sogar von jeder Injection, das ich beobachtete, wenn ich einen spitzen 
Kupferdraht durch die Mitte der Hornhaut bis in die linse einbohrte und so weit vorschob, dass er nicht mehr mit der Hornhaut in Berübrung war, sondern nur eine Strecke weit in die vordere Kammer vorragte. Ich kann vorläufig nur annehmen, dass durch die Anwesenheit einer reichlichen Menge von Eiweiss in der nächsten Umgebung des Fremdkörpers die geringe in jedem Augenblick zur Lösung kommende Menge von Kupfer sofort gebunden wurde und sonach nicht bis zu den gefässhaltigen Theilen, besonders der Iris, sich verbreiten konnte.

Ieh habe sehon vor einiger Zeit begonnen, die aus dem menschlichen Auge extrahirten oder dem frisch enucleirten Bulbus entnommenen Fremdlörper sammt dem sie umhüllenden Exsudat mit Hilfe der neueren Methoden auf das Vorhandensein von Spaltpilzen zu untersuchen und will über die bisher dabei erlangten Resultate hier kurz berichten.

\section{Fall 1.}

Dietrich Henke, 9 Jahre alt, verletzte sich vor 14 Tagen durch ein abgesprungenes Zündhutstück. $1 \mathrm{Mm}$. lange Hornhautnarbe am inneren oberen Rande, gegenüber kleine Lücke in der Iris. Beginnende Cataract. Fremdkörper nicht zu sehen. Nur leichte Injection. Selivermögen der Trübung entsprechend.

5 Monate später ist die Cataract completirt, die Kapsel nach innnen oben etwas verdickt, hier und nach aussen oben je eine hintere Synechie. Kaum merkliche Injection. Druck normal. Lichtsehein nicht sicher niederste Lampe, Projection gut. Modificirte Lincarextraction nach innen oben. Die zum Theil etwas gelblich gefärbte Cataract tritt leicht ans, aber kein Fremdkörper. Es wird jetzt noch etwas Iris gerade nach innen excidirt, und dadurch noch ein Stück verdickter Kapsel frei gelegt. Nach Extraction der letzteren mit der Kapselpincette folgt ein $3 \mathrm{Mm}$. langes, 11/2 $\mathrm{Mm}$. breites Kupferstückchen, das in der Gegend des Ciliarkörpers gesessen hatte, gefolgt von wenig normal consistentem Glaskörper.

Das Kupferstückchen zeigt auf einer Seite einen dünnen eitrigen Belag. Mikr. mohrkernige Eiterkörperchen mit 
blassen groben Köbnchen erfüllt. Ein mit Gentiana gefärbtes Trockenpräparat zeigt keine Spaltpilze. Keine Metallreaction, nur einige Pigmentzellen färben sich blau mit Blutlaugensalz und Salpetersänre (hämatogenes Pigment).

Die Linsensubstanz lässt zum Theil wohl erhaltene Linsenfasern erkennen, dazwischen viele grosse Körnchenzellen. Die Kapselverdickung besteht aus dicht gedrängten Zellen rerschiedener Gestalt, zum Theil ebenfalls Körnchenzellen.

Heilung normal mit Wiederherstellung won etwas Selvermögen, das später noch za bessern sein wird.

Mit grosser Wahrscheinlichkeit lässt sich somit für diesen Fall annehmen, dass das aseptiseh in die Gegend des Ciliarkörpers eingedrungene Kupferstückchen eine ganz umschriebene eitrige Entzündung hervorgerufen hatte, die sioh äusserlich fast gar nicht zu erkennen gab. Nach den oben mitgetheilten experimentellen Erfahrungen uber die Wirkung der Iris aufgelagerter Kupferstuckchen sind wir anch wohl berechtigt, in dem vorliegenden Falle die Entzündung der chemischen Wirkung des Fremdkörpers zuzuschreiben. Dasselbe gilt auch für die Fâlle, wo beim Menschen in der Umgebung eines auf der Iris sitzenden Kupferstückchens eine eitrige Exsudation beobachtet wird. Die Frscheinungen, welehe in den meisten Krankengeschichten berichtet werden, stimmen damit wohl ubberein, besonders auch darin, dass die Entzündung in der Regel keine sehr grosse Intensität und namentlich keine Neigung zur Weiterverbreitung besitzt.

Auch im folgenden Falle, wo ein auf der Iris sitzender Eisensplitter eine leichte umschriebene Exsudation bewirkt hatte, hat mich die Untersuchung in Bezug anf die Mitwirkung von niederen Organismen zu einem durchaus negativen Resultate gefuhrt.

Fall 2.

Karl Schmock, ein 17jähriger Schlosser, stollto sich vor, weil ihm 5 Tage rorher beim Arbeiten an der Drehbank ein Stahlsplitter in's Auge geflogen war. Leichte Schmerz- 
empfindung nur kurze Zeit nach der Verletzung. Mässige Ciliarinjeetion, Kammerwasser eben merklich getrübt, Iris leicht verfärbt. Nahe dem inneren unteren Hornhautrand eine kaum $1 \mathrm{Mm}$. lange Hornhautnarbe und gegenüber auf der Iris, etwas höher, ein ebenso grosses dottergelbes Exsudatklümpchen, das einen Fremdkörper einzuschliessen scheint. Annäherung eines Magneten bewirkt keine Verschiebung des Fremdkörpers. Glaskörper und Augengrund normal. $\quad s=\frac{20}{40}$.

Am folgenden Tag ist der Zustand unverändert. Nach Verengerung der Pupille durch Eserin liegt der Fremdkörper dem Pupillarrand der Iris ein wenig näher als dem Ciliarrande. Kleiner Linearschnitt nahe dem Hornhautrand mit schmalem Messer, Irisvorfall vermieden. Nach Einführung des Hirschberg'schen Electromagneten in die Wunde sieht man den Fremdkörper langsam vorrücken und in der Wunde erscheinen, dahinter aber die damit verklebte Iris. Ersterer lässt sich mit dem stumpfen Häkchen leicht von der Iris lösen, die alsdann mit dem Spatel reponirt wird, worauf in 8-10 Tagen. ganz befriedigende Heilung erfolgt.

Das extrahirte Stüekchen erwies sich als ein flacher Stahlsplitter von $1 \frac{1}{4} \mathrm{Mm}$. Länge, $1 \mathrm{Mm}$. Breite und 1/4 $\mathrm{Mm}$. Dicke und von unregelmässig dreieckiger Gestalt. Nur die vordere, nach dor Hornhaut gekehrte Flä.che war von der schon in situ bemerkten, eigelben Exsudatschicht bedeckt, während die der Iris zugokehrte, hintere Fläche eine schwarze Farbe darbot.

Die Exsudatschicht war nur von geringer Dicke und bem stand, wie die sofort vorgenommene Untersuchung ergab, aus mässig zellenreichem Fibrin, welches durch die Einwirkung des Eisens in besonderer Weise verändert war und durch Einlagerung von fein vertheiltem Eisenoxydhydrat eine gelbe Färbung angenommen hatte. Die Fibrinfäden sind eigenthümlich steif, stärker lichtbrechend und in kürzere und längere Stiicke getheilt, in welche sie bei der Präparation auch leicht aus einander brechen. In das Netz dieser Fäden sind stark lichtbrechende, gelbbraun gefärbte, undeutlich grobkörnige Zellen von der Grösse der Lympbkörperchen und darüber eingelagert. Thre Färbung ist meistens ziemlich gleichmässig, doch finden sich bei einzelnen auch dunkler rostfarbige Körner und Klümpchen eingeschlossen. Das Gewebe ist ziemlich opak, wird aber durch Essigsüure etwas gehellt, so dass durch 
Hämatoxylin die Kerne der mit Eisen imprägnirten Zellkörper sichtbar werden. Znsatz von gelbem Blutlaugensalz und Salpetersäure bewirkt eine böchst intensive Blaufärbung dos ganzen Gewebos. Das Verhalten ist genau dasselbe, wie ich es nach Einführung von Nahnadelstucken in den Glaskörper am Kaninchenauge oft genug beobachtet habe und stimmt in chemischer Beziehung auch durchaus mit dem Verhalten der Linse bei Anwesenheit von Eisonstucken in derselben überein. *) Anf die Anwesenheit von Spaltpilzen wurde sowoll frisch als nach Zusatz von rerschiedenen Reagentien und mit Hilfo Fon Farbungsmitteln, aber mit durchaus negativen Resultat untersucht. Uebrigens ist nach der ziemlich intensiven chemischen Wirkung, welche der Stahlsplitter auf seine Lmgebung ansgeübt hatte, deren Vorkommen gewiss auch nicht zu erwarten.

Wir werden uns den Hergang also so vorstellen dürfey, dass beim Menschen auch die chemische Wirkung des Eisens an der Berührungsstelle mit der Iris stark genng ist, um hier eine umschriebene Entzindung za bewirken. Das dadurch gelieferte Exsudat wird dann weiterlain durch dieselbe chemische Wirkung in eigenthürnlicher Weise verändert, so dass es die im obigen Falle beschriebene Beschaffenheit annimmt. Anch das Eisen ist somit für das menschliche Auge als eine an und für sich Entzündung erregende Substanz zu betrachten. Die dadurch bewirkte Entzündung ist aber ziemlich geringen Grades, jedoch stärker als beim Kaninchen-Auge. Abgesehen von diesem graduellen Untersehiede sind aber die Frscheinungen im wesentlichen dieselben, so dass die Thatsachen unter einander in ganz befriedigender Weise harmoniren.

Was die gar nicht seltenen Falle betrifft, wo nach Eindringen von Metall-, besonders Kupfersplittern in das Bereich des Ciliarkörpers oder der Chorioidea eine ausgedehntere eitrige Glaskörper-Infiltration beobachtet wird,

*) Vergl. unten S. 256-258. 
so möchte ich mir zur Zeit über die Möglichkeit der aseptischen Entstehung derselben noch kein bestimmtes Urtheil erlauben. In nachstehendem Falle hat sich sowohl bei mikroskopischer Untersuchung mit Hilfe von Tinctionsmitteln, als nach Impfung in die vordere Augenkammer, (die keine Entzündung hervorrief), ein negatives Resultat ergeben; doch würde ich zur völligen Sicherheit für gerathen halten, mit den Entzündungsprodukten Culturversuche auf geeigneten Nährsubstanzen anzustellen, was ich vorkommenden Falles auszuführen gedenke.

\section{Fall 3.}

Wilhelm Sasse, 3 Jahre alt, verletzte sich vor 4 Wochen in der Sehlosserwerkstätte durch Hämmern auf einen Amboss. Auge mässig, aber tief injicirt. Kleine lineare Hornhautnarbe nach unten mit Einklemmung der Iris. Dahinter eine strangförmige Linsentrübung, welche von dem etwas eingerissenen unteren Pupillenrand die Linse durchsetzt. Ausgedehnte, goldgelb schimmernde Infiltration im unteren Theil des Glaskörpers. Pupille nach oben schwarz. Druck etwas herabgesetzt. Von Fremokörper nichts zu sehen.

Anatomische Untersuchung des sofort enucleirten, frischen Auges. Beim Aufschneiden im verticalen Meridian fliesst viel gelbliche, nur leicht trübe Flüssigkeit ans, die beim Stehen ein gallertiges Gerinnsel ausscheidet. Mit Essigsäure giebt sie nur mässige Trübung, mit Salpetersäure einen dicken käsigen Niederschlag. Nach der Gerinnung enthält sie nur ziemlich spärliche Lymphkörperchen.

Die eitrige Infiltration nimmt einen grossen Theil der unteren Hälfte des Glaskörpers ein. Der übrige Glaskörper ist geschrumpft und von der Retina abgelöst, der dadurch entstandene Raum war von der ausgelaufenen Flüssigkeit erfüllt gewesen. Dicht hinter der Linse erstreckt sich die Trübung noch auf den oberen Theil des Glaskörpers, ist aber hier weniger intensiv, mehr von gravlichem Aussehen. Die strangförmige Trübung der Linse setzt sich in eine schalenförmige, dicht unter der Kapsel liegende Trübung der hinteren Corticalls fort. Der grösste Theil der Linse ist durchsichtig.

Die Papille und angrenzende Retina sind etwas verdickt; 
die Retina zart und mit Ausnahme einer kleinen zadiaren Falte ibberall anliegend.

Der Tremdkörper sitzt fast genau im rerticalen Meridian dicht hinter dem Aequator nach unten den Augenhäuten auf, in Exsudat eingehüllt. Dahinter sieht man anf der Retina eine grössere Zahl hirsekorngrosser Exsudatknötchen, die sich leicht abheben lassen. Der Glaskorper ist an dieser Stelle abgelost. Beim Einschneiden der Exsudathülle kommt ein $31 / 2 \mathrm{Mm}$. langer, $1^{1 / 2} \mathrm{Mm}$. breiter Eisensplitter zum. Vorschein. Das ibn zunächst umgebende Exsudat ist gelbbraun gefärbt und besteht aus zarten amorphen Membranen und Fetzen, dio starke Fisenreaction geben; daneben finden sich viele fettartig glänzende Kömchen; weiterhin Eiterkörperchen, die dicht mit glanzenden Tröpfchen erfullt sind, und vereinzelt dunkelbraune Pigmentkörner und Klumpen, die ebenfalls Eisenreaction geben. Der Fremdkörper sitzt der Chorioidea auf, die Retina ist an der Stelle als solche nicht mehr zu erkemen. Die Eiterkörperchen im Glaskörper enthalten ebenfalls fettartig glïnzende Tröpfchen, die aber mit Osmiumsäure sich nicht schwärzen und mit Fuchsin nicht roth fürben, also wohl nicht ans Fett bestehen; der weniger getrübte Theil des Glaskörpers zeigt ein diehtes Fibrinnetz mit mehr vereinzelten Eiterkörperchen. Mikrocokken oder sonstige parasitare Elemente auch mit Tinetion nicht nachzumeisen.

Von dem eitrig infiltrirten Glaskörper wurde sofort nach Erüfinung des Anges je ein ziemlich grosses Stückchen mit vorher geglïhten Instrumenten einem Kaninchen an beiden Augen dureh einen kleinen Lanzonschnitt in die vordere Kammer gebracht. Ausser etwas Fibringerinuung in der Ungebung des Fremdkörpers, wie sie anch bei ganz indifferenten orgam nischen Substanzen stets einzutreten pflegt, blieb der Eingriff ohne weitere Folgen und nach 19 Tagen waren die Stückchen spurlos resorbirt.

Trotz dieser negativen Resultate möchte ich, wie schon bemerlkt, diesen Fall noch nicht für völlig beweisend halten und weitere Erfahrungen abwarten.

Auch in der Hornhaut sitzende Eisenstuckchen scheinen durch ihre chemische Wirkung allein Entzündung hervorzurufen, wie aus folgender Beobachtung hervorgeht, 
die ich noch während der Abfassung dieser Zeilen zu machen Gelegenheit hatte.

\section{Fall 4.}

Einem Arbeiter war for $1^{1 / 2}$ Tagen ein Stahlfunken auf die Hornbaut geflogen. Man bemerkt etwas nach unten von der Hornhantmitte einen dunklen, leicht prominirenden Punkt, der von einem schmalen gelben Saum umgeben ist, das Ganze von nur ca. $1 / 2 \mathrm{Mm}$. Durchmesser. Die Umgebung zeigt eine leichte diffuse Trübung in der Ausdehnung ron mehreres Millimetern, am Boden der Kammer ein eben merkliches Hypopyon. Bei der Entfernung des kleinen Fremdkörpers zeigt sich, dass er sich etwas tiefer als gewöhnlich in die Hornhaut eingebrannt hatte. Die Untersuchung des gleichzeitig entfernten Stückrob :- decrotischen Hornhautgewebes erwies dasunve, braun gefärbte Hornhautgrundsubstanz, die ente sehr intensive Eisenreaction gab. Die vom Rande herrührenden Partikelchen zeigten mit der Färbungsmethode (Gentianaviolett) ganz gut erhaltene Epithelzellen, aber keine Spur von Spaltpilzen, welche sonst bei Beginn der Keratitis septica durch diese Methode stets mit Leichtigkeit zu erkennen sind.

Nach Entfernung des Fremdkörpers war das Hypopyon schon am nächsten Tage verschwunden und erfolgte in Kurzem vollständig $\ominus$ Heilung.

Es ist bekannt, dass Stahlfunken, die etwas länger als gewöhnlich auf der Hornhaut sitzen bleiben, daselbst eine leichte demarkirende Entzündung erregen, die zur spontanen Abstossung des Fremdkörpers führt und sich auch wohl mit einem leichten Hypopyon verbinden kam; in obigem Fall trat diese Entzündung entsprechend dem tieferen Sitze des Fremdkörpers relativ früh ein. Dass sie durch die chemisehe Wirkung des Fremdkörpers bedingt ist, scheint auch daraus hervorzugehen, dass sie auftritt, noch ehe es zur Elimination des letzteren gekommen ist, wo also der kleine Schorf noch festsitzt und ein Eindringen von Infectionskeimen wohl kaum gestattet. In gleichem Sinne argumentirt die rasche Heilung nach 
Entfernung des Fremdkörpers, wie sie bekanntlich anch sonst in derartigen Fallen einzutreten ptlegt.

Es würde sich verlohnen, auch in anderen Fallen lieser Art, die sich in der Praxis ja oft genug darbieten, die oben mitgetheilte Untersuchung za wiederholen, da bei der minimalen Menge des zur mikroskopischen Untersuchung zu gewinnenden Materials nur durch Untersuchung einer gewissen Zabl von Fallen Irrthumer sicher auszuschliessen sind.

Auch für das Ausbleiben irgend nennenswerther entzündlicher Reaction bei Sitz des Fremdkörpers anf der Linsenkapsel kann ich eine neue Beobaehtung beim menschlichen Ange als Belag anführen, die .. $e^{* m}$ foïher von Landmann*) mitgetheilte aus der u.. o anreiht und in vollkommenem Einklang mit den oben su schilderten experimentellen Ergebnissen steht.

\section{Fall 5.}

Simon Christians, 18 Jahre alt, giebt an, dass ihn ror $3 / 4$ Jahr beim Hämmern auf Eisen etwas ins Auge geflogen sei. - Leichte Röthung und Thrünen des Auges verloren sich schon nach wenigen Tagen, so dass Patient auf eine etwaige Sehstörung nicht weiter achtete und erst vor 3 Monaten die inzwischen erfolgte Erblindung dieses Auges wahrnahm.

Kleine lineare Narbe in der Hornhautmitte. Iris grünlich verfärbt (die andere hellblau); Pupillarrand frei; Linse vollm ständig getrubt und etwas geschrumpft. Etwas oberhalb der Mitte der Pupille eine kleine Kapselnarbe, deren Rïnder, wie auch der dahinter liegende Theil der Cataract, eine gelbliche Farbe darbieten, während die Randtheile melrr weisslich aussehen. Nach Atropinisirung sieht man dem unteren Theil der Linsenkapsel einen schmalen, senkrecht gerichtoten Fremdkörper anfgelagert, dessen unterer Rand sich noch etwas hinter der Tris verbirgt, von braunex Farbe, am Rande mit einom gelblichen Streifen. Beide Augen leicht katarrbalisch injicirt, das verletzte nicht wesentlich mehr als das andere. Nodificirte linear-Extraction nach unten. Nach der Iris-Fxcision lässt

*) Dieses Archiv XXVIII. 2, S. 188, Fall 51. 
sich der Fremdkörper, ein $3 \mathrm{Mm}$. langes, kaum 1/2 Mm. breites, spitzes Eisenstückchen mit der magnetisch gemachten Kapselpincette leicht extrahiren, worauf die kleisterartig erweichte Corticalis glatt und vollständig ausgestrichen wird.

Heilung normal mit gutem Sehvermögen.

Der gelbliche Belag des Splitters besteht aus amorphen Partikelchen, die starke Eisenreaction geben. Der gelblich gefärbte Theil der Cataract ist etwas compacter als der übrige, erscheint mikroscopisch von blass olivenbrauner Farbe, zeigt wohl erhaltene blass-feinkörnige Linsenfasern, die wie in der Norm beisammen liegen and ebenfalls starke Eisenreaction geben. In der übrigen Linsensubstanz finden sich zwischen den Fasern zahlreiche Myelintröpfchen, stellenweise auch grosse mit glänzenden Tröpfchen erfültte Zellen, wie sie auch sonst bei traumatischer Cataract vorkommen.

Der Fremdkörper hatte in diesem Falle die Linsenkapsel im Centrum verletzt, war auf derselben liegen geblieben und scheint sich erst nachträglich etwas gesenkt zn haven. Der Mangel einer hinteren Synechie spricht dafür, dass er schon anfangs nicht in direkte Berührung mit der Iris kam, won welcher er nun durch die Schrumpfung der linse etwas entfernt gehalten wurde: Der Fall zeigt, dass auch bei Sitz eines Eisensplitters auf der Linsenkapsel, nicht nur im Inneren der Linse, ein ganz reizloser Zustand vorkommt. Er bestätigt zugleich die Richtigkeit meiner auf Thierversuchen beruhenden Angabe*), dass die orangegelbe Farbe der Linse bei Gegenwart von Eisensplittern von Eisenoxydhydrat herrührt.

$\mathrm{Zu}$ derselben Beobachtung wurde mir später noch in einem anderen Falle beim Menschen die Gelegenheit geboten.

\section{Fall 6.}

Es handelt sich um eine von mir durch einfachen Linearschnitt extrahirte traumatische Cataract, welche einen kleinen Eisensplitter von ca. $1 \mathrm{Mm}$. Durchmesser einschloss. Diejenigen

*) Th. Leber, Internat, med. Congr. Transact. Vol. III und Landmann, dieses Arch. XXVIII. 2, S. 195.

v. Graefe's Archir für Ophthalmologie, XXX, 1. 
Theile der Cataract, welche den Fremdkörper zunächst umgaben, hatten eine auffallend brännliche Farbe und zeigten wohl erhaltene, leicht körnig getrübte Linsenfasern; weiterhin verlor sich die Färbung allmälig. Die braun gefärbten Partien gaben eine höchst intensive blaue Färbung mit gelbem Blutlaugensalz und Salpetersäure, die übrige Substanz dieselbe Reaction, nur ziemlich schwach, anch da, wo keine deutliche gelbe Färbung mehr zu erkennen war. (Heilung normal mit gutem Sehvermögen.)

Meine Beobachtungen bestätigen also durchaus die bisher giltige Annahme, dass Fremdkörper aus oxydablen Metallen für sich allein entzündungserregend wirken können, zeigen aber zugleich, dass sie nicht unter allen Umständen entzündungserregend wirken müssen, indem die Wirkung nicht nur von der Art des Metalls, sondern auch von dem Sitz des Fremdkörpers sehr wesentlich abhängig ist. 